\section{IN BRIEF}

\section{$\Rightarrow$ PROSTATE CANCER}

\section{Genomic correlates of clinical outcomes}

Comprehensive genomic and transcriptomic analysis of 429 patients with metastatic castration-resistant prostate cancer (mCRPC) has been linked with histology and clinical outcomes to investigate heterogeneity in the genomic landscape of metastatic prostate cancer. Of the genomic alterations examined, which included alterations in $A R, R B 1$ and TP53, only RB1 alteration was significantly associated with poor survival. Alterations in RB1, AR and TP53 were associated with shorter time on androgen receptor signalling inhibitor treatment. The study identifies molecularly defined groups of patients who might benefit from an aggressive treatment approach. The authors have made the tumour-level and patient-level data available for further research.

ORIGINAL ARTICLE Abida, W. et al. Genomic correlates of clinical outcome in advanced prostate cancer. Proc. Natl Acad. Sci. USA https://doi.org/10.1073/pnas.1902651116 (2019)

\section{TESTICULAR CANCER}

\section{Understanding obesity interactions with germ cell tumours}

Obesity is associated with better outcomes in some metastatic cancers, for example, renal cancer, but the association between BMI and germ cell tumour (GCT) outcomes has not been reported. Medical records of 1,161 patients with GCT who were treated at Dana-Farber Cancer Institute between 1997 and 2012 were reviewed and logistic regression analysis used to investigate the association between $\mathrm{BMI}$ and clinical features of GCT. In men with stage $1 \mathrm{GCT}, \mathrm{BMI} \geq 25$ was not associated with an increased risk of relapse compared with those with $\mathrm{BMI}<25$. In metastatic disease, men with $\mathrm{BMI}<25$ were less likely to present with good-risk disease, but BMI was not associated with risk of relapse. Overall, men with lower BMI are more likely to present with intermediate-risk or poor-risk GCT.

ORIGINAL ARTICLE McGregor, B. A. et al. Body mass index and outcomes in germ cell tumors. Clin. Genitourin. Cancer https://doi.org/10.1016/j.clgc.2019.04.012 (2019)

\section{$\Rightarrow$ INFECTION}

\section{No gentamicin for first-line gonorrhoea therapy}

Ceftriaxone is the current first-line treatment for gonorrhoea; however, antibiotic resistance is emerging and new approaches are needed. The G-ToG study was a multicentre, parallel-group, randomized noninferiority trial that compared treatment with gentamicin with ceftriaxone for gonorrhoea at 14 sexual health clinics in England. Patients received either $240 \mathrm{mg}$ of gentamicin or $500 \mathrm{mg}$ of ceftriaxone as a single intramuscular injection plus $1 \mathrm{~g}$ of oral azithromycin. In total, 720 participants ( $81 \%$ male) were randomized, 358 in the gentamicin group and 362 in the ceftriaxone group. Clearance of genital infection was similar in the two groups ( $94 \%$ in the gentamicin group and $98 \%$ in the ceftriaxone group), but clearance of pharyngeal infection and rectal infection was lower in the gentamicin group ( $80 \%$ versus $96 \%$ and $90 \%$ versus $98 \%$, respectively). Overall, the trial was unable to demonstrate noninferiority of gentamicin compared with ceftriaxone in the clearance of gonorrhoea at all infected sites. Furthermore, gentamicin was associated with more severe pain at the injection site and higher costs.

ORIGINAL ARTICLE Ross, J. D. C. et al. Gentamicin compared with ceftriaxone for the treatment of gonorrhoea (G-ToG): a randomised non-inferiority trial. Lancet https:// doi.org/10.1016/S0140-6736(18)32817-4 (2019)

\title{
Mechanisms of high-dose testosterone
}

A new study describes the characterization of the mechanisms underlying responses to supraphysiological testosterone therapy (SPT) for men with castration-resistant prostate cancer (CRPC). The findings in patient-derived xenografts (PDXs) show that SPT results in sustained suppression of AR-V7 and E2F signalling and the DNA damage response (DDR) in responders, describing potential mechanisms of tumour growth inhibition by SPT.

SPT results in sustained suppression of AR-V7 and E2F signalling and the DNA damage response

go

Previous studies have shown that treatment of CRPC with SPT can be effective in some patients. "Despite preclinical results showing inhibition of prostate cell proliferation by SPT and positive results from clinical trials, use of SPT in advanced prostate cancer is still highly debated by clinicians," explains senior author Eva Corey from the University of Washington. "However, patients failing enzalutamide have very limited treatment options, and SPT presents an option for durable response and improved quality of life for some of these men." Thus, Corey's team set out to study mechanisms of SPT action and to discover biomarkers to identify those patients who respond.

Using advanced prostate cancer PDXs in mice, the team identified four PDXs that responded to SPT with tumour growth inhibition. These PDXs endogenously had lower proliferation scores and E2F signalling scores but higher androgen receptor $(A R)$ mRNA levels, higher AR activity score and higher AR-V7 junction reads than PDXs that did not respond.
The team then explored SPT response in three CRPC PDXs that were resistant to enzalutamide. These models had varied responses to SPT: one PDX showed durable, the second transient and the third no growth suppression. In all three models during SPT, $A R$ and $A R V 7$ expression was downregulated but AR target gene expression was upregulated. By contrast, AR-V7 target gene expression was downregulated in SPT-responding PDXs only and was restored when the transiently responding PDXs became SPT resistant. Furthermore, the MYC-E2F pathway and DNA replication and DDR programmes were repressed in the responding PDX models and were also restored in the transient responder upon SPT resistance, indicating the mechanism of growth inhibition caused by SPT.

"This first molecular insight into SPT response and resistance together with a clinical case report provide evidence to nominate patients with DDR deficiency as remarkable and durable SPT responders," comments Corey. "Furthermore, our data showed that enzalutamide rechallenge upon SPT resistance resulted in PSA level decreases but did not inhibit tumour progression, highlighting the need for monitoring radiographic progression and not relying on PSA levels to make conclusions about SPT efficacy."

The team is now working to identify co-drivers of the observed DDR pathway inhibition for therapeutic targeting to augment SPT effectiveness. They are also investigating AR signalling differences between SPT responders and non-responders to identify biomarkers for patient selection.

Clemens Thoma

ORIGINAL ARTICLE Lam, H.-M. et al. Durable response of enzalutamide-resistant prostate cancer to supraphysiological testosterone is associated with a multifaceted growth suppression and impaired DNA damage response transcriptomic program in patient-derived xenografts. Eur.Urol. https://doi.org/10.1016/ j.eururo.2019.05.042 (2019) 